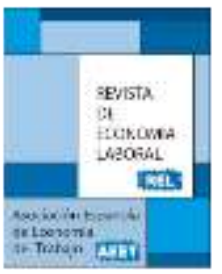

\title{
PROTECCIÓN DEL EMPLEO Y FLUJOS DEL MERCADO DE TRABAJO EN ESPAÑA: EFECTOS DE LAS REFORMAS DE 2010 Y $2012^{1}$
}

\author{
Ángel Luis Gómez Jiménez ${ }^{2}$ \\ Universidad de Alcalá y Banco de España
}

Aceptado Marzo 2016

\section{Resumen}

En este artículo se analiza el impacto de las reformas laborales de 2010 y 2012 en España, particularmente en lo que atañe a la legislación de protección del empleo, sobre los flujos entre el desempleo y el empleo asalariado (y dentro de este) con datos de la Encuesta de Población Activa. En el área de la protección del empleo, las reformas se reflejan en una disminución del grado de protección de los contratos indefinidos contra los despidos, tanto individuales como colectivos, al tiempo que se ha seguido facilitando la contratación temporal. En conjunto, dichas reformas no parecen haber tenido en sus primeros años de aplicación efectos sustanciales sobre las probabilidades de contratación ni de separación del empleo, aunque sí habrían incrementado la tasa de rotación de los contratos temporales, con un aumento de las salidas de este tipo de empleo.

Palabras clave: Protección del empleo, flujos de creación y destrucción de empleo, reformas laborales, contratación, despido, temporalidad.

Clasificación JEL: J08, J21, J63

\section{Abstract}

This article analyzes the impact of the 2010 and 2012 labour reforms in Spain, particularly in those aspects referred to the employment protection legislation (EPL), on the flows between unemployment and dependent employment (including job-to-job flows) using Labour Force Survey data. Concerning to the EPL area, the reforms result in a decrease in the level of the permanent employment protection against both single and mass dismissals, while fixed-term hiring is still being eased. Overall, in their early stages such reforms don't seem to have affected substantially the hiring or separation rates, although they would have increased the turnover ratio of the fixed-term workers, due to a rise in the outflows of this kind of employment.

Key words:Employment protection, employment inflows and outflows, labour reforms, hiring, dismissal, temporary ratio.

JEL Classification:J08, J21, J63

${ }^{1}$ El autor agradece a Carlos García-Serrano y José María Arranz su tutoría del Trabajo Fin de Máster en Análisis Económico Aplicado, impartido por la Universidad de Alcalá, en el que se basa este artículo, así como los comentarios de dos evaluadores anónimos. Las opiniones y análisis expresados en este artículo son responsabilidad del autor y, por tanto, no comprometen al Banco de España ni a la Universidad de Alcalá.

${ }^{2}$ Autor para correspondencia:algomez@bde.es.

C Revista de Economía Laboral 


\section{Introducción}

Desde el trabajo seminal de Lazear (1990), una literatura muy extensa se ha dedicado a explorar, tanto teórica como empíricamente, el impacto de la legislación de protección del empleo sobre los resultados del mercado de trabajo, tanto en términos de nivel y dinámica del empleo y del desempleo, como de duración de los episodios de desempleo, efectos sobre los salarios, sobre la productividad y la innovación, creación y destrucción de empresas, composición del empleo, dualidad e interacción con otras instituciones del mercado de trabajo. En el análisis empírico se han usado habitualmente indicadores que permitan las comparaciones internacionales de los niveles de protección del empleo, siendo los que elabora la OCDE los de uso más extendido. De acuerdo con estos indicadores, durante la última década se aprecia una tendencia a nivel internacional a la reducción de la protección del empleo, centrada en los despidos individuales y colectivos. Entre 2008 y 2013 más de un tercio de los países de la OCDE (principalmente aquellos que encabezaban el ranking al principio del período) emprendieron alguna reforma en ese sentido, lo que contrasta con lo ocurrido durante los años 90, cuando también se observó una cierta convergencia internacional en el grado de protección del empleo, pero como consecuencia de los cambios en la regulación de los contratos temporales (OCDE, 2006; OCDE, 2013a).

$\mathrm{Al}$ abordar cualquier evaluación de los efectos de las reformas, se ha de tener en cuenta que el análisis del mercado de trabajo en términos de stocks (de empleo, desempleo, activos, vacantes, etc.) es insuficiente para apreciar la heterogeneidad de su respuesta en términos de las características del trabajador, del tipo de contrato o del sector de actividad (Pissarides, 2000; Davis, Faberman y Haltiwanger, 2006). Sin embargo, un enfoque desde la perspectiva de los flujos brutos de creación y destrucción de empleo tiene en cuenta toda la variabilidad que se desprende de las transiciones entre los tres posibles estados en que puede hallarse un individuo (empleo, desempleo e inactividad) y permite identificar mejor el papel desempeñado por la configuración institucional del mercado de trabajo. La literatura empírica acerca de los efectos de la legislación de protección del empleo (y de sus reformas) sobre los flujos del empleo y del desempleo y la rotación laboral ofrece resultados dispares (Bertola et al., 1999; Addison y Teixeira, 2003; OCDE, 2006; Boeri, 2011; Bassanini y Garnero, 2013), si bien se aprecia cierto consenso respecto al impacto amortiguador de la regulación del despido sobre los flujos de entrada y salida del desempleo. En este 
sentido, algunos análisis señalan que las últimas reformas laborales en España han aumentado la rotación laboral y la dualidad del mercado de trabajo (Pérez Infante, 2015).

Estas reformas han modificado algunos de los aspectos más relevantes del marco institucional en el que se desenvuelve el entramado de relaciones laborales, como son los que afectan a las modalidades de contratación, a los agentes de la intermediación laboral, al sistema de negociación colectiva y a los costes y mecanismos de despido. Entre los principales objetivos con los que se plantearon dichas reformas figuran precisamente la corrección de la dualidad que caracteriza el mercado de trabajo español y la consecución de la flexibilidad interna necesaria para que el ajuste del mercado de trabajo no siga recayendo sobre el empleo, especialmente el temporal.

En cuanto a la evaluación de los efectos de las reforma de 2012 sobre el empleo, en Ministerio de Empleo y Seguridad Social (2013) se estiman funciones de empleo con datos trimestrales ${ }^{3}$ del período 1980 1T-2012 1T, a partir de las que se realizan previsiones para los 4 trimestres posteriores a la reforma. El resultado se compara con el empleo observado para este periodo de predicción y la diferencia se atribuye a la reforma, acumulándose un resultado positivo de $180 \mathrm{mil}$ asalariados en el sector privado. De forma análoga, Izquierdo et al. (2013) utilizan los residuos de una ley de Okun sobre los flujos de salida del empleo al paro y de entrada al empleo desde el desempleo, con datos de la estadística de flujos de la EPA y no encuentran efectos significativos de la reforma de 2012 ni sobre el volumen de flujos ni sobre la ratio de temporalidad. Finalmente, en OCDE (2013b) se estiman regresiones de discontinuidad ${ }^{4}$ sobre diversas variables flujo: tasas de contratación obtenidas de los microdatos de la EPA (2008 1T2013 2T), nuevos contratos indefinidos según las estadísticas del Servicio Público de Empleo Estatal (SEPE) para el período Enero 2006Julio 2013, tasas de pérdida de empleo a nivel de establecimiento calculadas a partir de la información de la Encuesta de Coyuntura Laboral (2006-2012), despidos individuales y colectivos que se desprenden de la estadística de prestaciones por desempleo (Enero

\footnotetext{
${ }^{3}$ No se indica la fuente de la que proceden estos datos.

${ }^{4}$ Se controla por la tasa de desempleo estandarizada, afiliados y tendencia temporal polinómica y se realizan controles de robustez con medidas alternativas del ciclo económico.
} 
2007-Julio 2013) y transiciones del desempleo al empleo según la Muestra Continua de Vidas Laborales (2003-2012). Entre los efectos de la reforma que resultan de dichas estimaciones destacan los siguientes: un incremento del 18\% en la contratación indefinida a tiempo completo, mayor movilidad entre tipos de contrato, una moderación de las salidas del empleo temporal y una reducción de los despidos colectivos. Además, el impacto positivo sobre la contratación estaría siendo mayor en las empresas pequeñas.

El propósito de este artículo es analizar el impacto de las reformas laborales de 2010 y 2012, centrándose, a diferencia de los estudios mencionados, en lo que atañe a la legislación de protección del empleo, sobre los principales flujos del mercado de trabajo (y no solo entre el desempleo y el empleo asalariado, sino también las transiciones dentro de este). Para ello, se utilizan las estadísticas de la Encuesta de Población Activa (EPA) con datos hasta el primer trimestre de 2015. Ha de tenerse en cuenta que el análisis de los efectos de las reformas, más allá del entorno cíclico adverso en que estos se producen, se halla sujeto a limitaciones importantes. Se trata de reformas de carácter estructural, que afectan a múltiples aspectos de las relaciones laborales, lo que, por una parte, impide identificar un grupo de control bien definido, y por otra, requiere un análisis integral que tenga en cuenta la interacción de todos los elementos del mercado de trabajo. Además, las reformas conviven con otras muchas medidas de política económica que sin duda, habrán afectado a las principales variables del mercado de trabajo, de manera más o menos directa, entre las que podemos citar como más relevantes las sucesivas reformas de las pensiones o del sistema de protección por desempleo.

El artículo se organiza como sigue: en la segunda sección se presentan brevemente los indicadores de protección del empleo que elabora la OCDE y los resultados que arrojan para España. En la sección tercera se presentan los datos y se describen los flujos del empleo asalariado y su evolución desde el año 2005. La cuarta sección presenta la metodología seguida en el análisis empírico y los resultados de diversos modelos estimados al objeto de valorar la incidencia de ambas reformas sobre los procesos de creación y destrucción de empleo. Finalmente, se resumen las principales conclusiones. 


\section{El indicador de protección del empleo de la OCDE: resultados para España}

El indicador de la OCDE se compone de 21 ítems básicos, clasificados en tres áreas principales: despidos individuales de trabajadores con contrato indefinido, empleo temporal y regulación adicional de los despidos colectivos (cuadro 1). En http://www.oecd.org/els/emp/EPL-Methodology.pdf se presenta el procedimiento de asignación de valores a estos componentes básicos, en una escala cardinal que va de 0 a 6 , con los valores más altos indicando mayor restricción. Los indicadores sintéticos se obtienen con las ponderaciones recogidas en el cuadro 1. Además, antes de la actualización de 2013, se obtuvieron tres versiones del indicador global ${ }^{5}$ :

- La versión 1 es una media simple de los indicadores de contratos indefinidos y temporales, sin incluir los tres nuevos ítems $(9,16$ y 17) incorporados en la actualización de 2008. Las series temporales de esta versión comienzan en 1985.

- La versión 2 es la media ponderada de los dos indicadores anteriores y el de despidos colectivos. Las series temporales de esta versión arrancan en 1998.

- $\quad$ La versión 3 añade, desde 2008 los ítems 9, 16 y 17.

${ }^{5}$ La actualización de 2013 no incluye un indicador global, ya que se considera que no es neutral flexibilizar el mercado de trabajo mediante la relajación del despido de los trabajadores indefinidos o la contratación de los trabajadores temporales. 


\section{Cuadro 1. Componentes básicos y ponderaciones para el cálculo de los indicadores sintéticos de} protección del empleo.

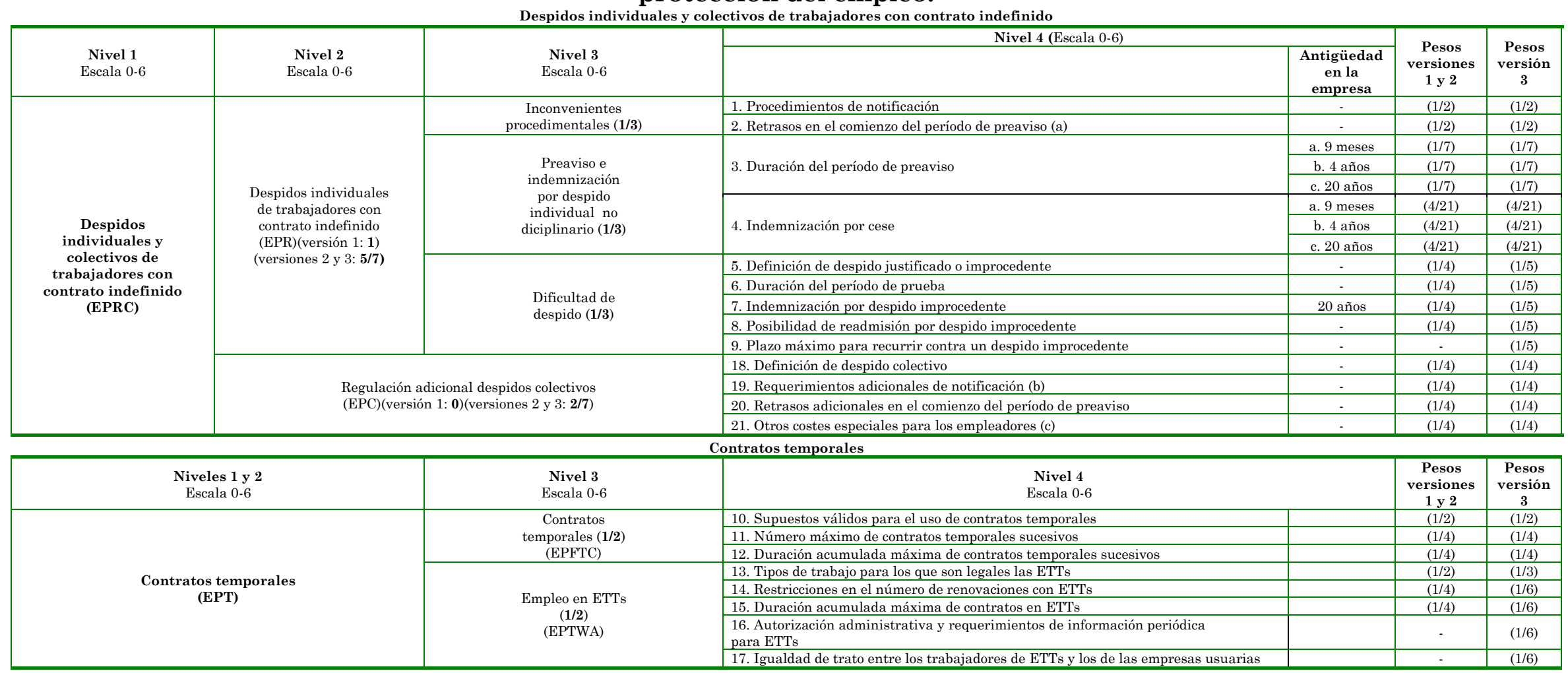

Fuente: http://www.oecd.org/els/emp/EPL-Methodology.pdf

a. Donde sea relevante se incluyen los siguientes supuestos: 1 día cuando el despido se pueda comunicar verbalmente o la notificación se pueda entregar directamente al empleado, 2 días cuando se deba utilizar el correo ordinario, 3 días cuando se deba utilizar el correo certificado y 6 días cuando se requiera procedimiento de aviso.

b. Al comité de empresa (o los representantes de los trabajadores) y autoridades.

c. Indemnizaciones adicionales por cese y planes sociales de compensación (recolocaciones, nueva formación, etc.). 
Los gráficos 1 y 2 presentan la serie temporal 1985-2013 del indicador de protección del empleo de la OCDE (versión 1) para los despidos individuales y los contratos temporales en España. Se observa que tanto en el caso de los contratos indefinidos como en el de los temporales, España ha seguido una clara tendencia a la desregulación durante los últimos 30 años, marcada por las sucesivas reformas laborales, particularmente las de 1994, 2010 y 2012 en el primer caso y estas mismas, más la de 2006, en el segundo. El gráfico 3 muestra que si se tienen en cuenta las regulaciones adicionales para los despidos colectivos se mantiene lo observado para los despidos individuales, ya que estas solo se habrían modificado en la reforma de 2012, en su conjunto, a lo largo de este período.

\section{Gráfico 1. España: protección de los contratos indefinidos contra despidos individuales. Versión 1 del indicador a 1 de Enero.}

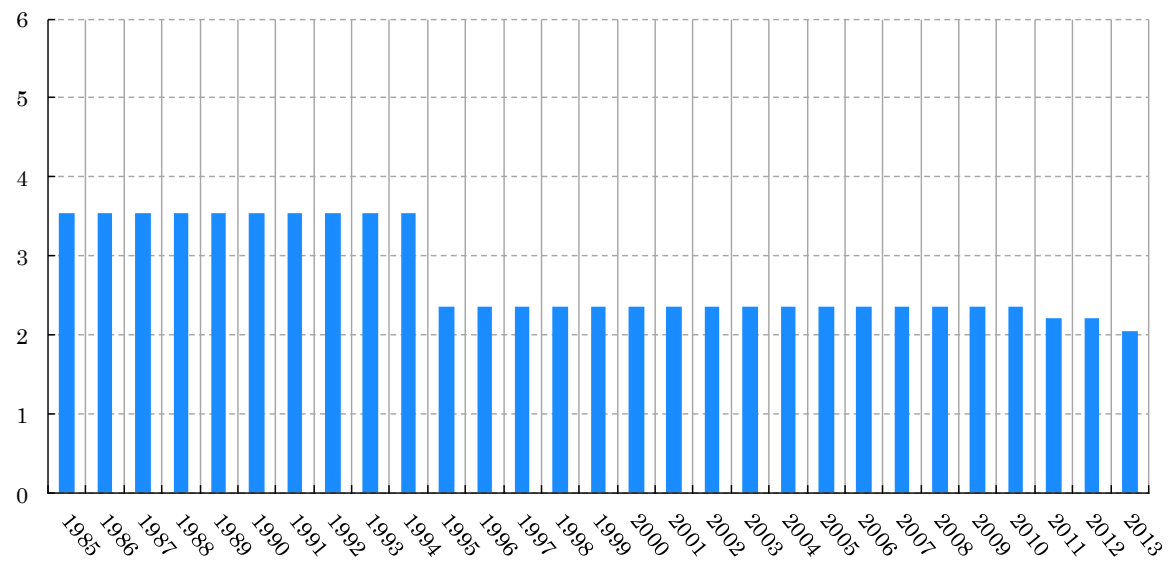

Fuente: OCDE. 
Gráfico 2. España: grado de restricción en los contratos temporales individuales. Versión 1 del indicador a 1 de Enero.

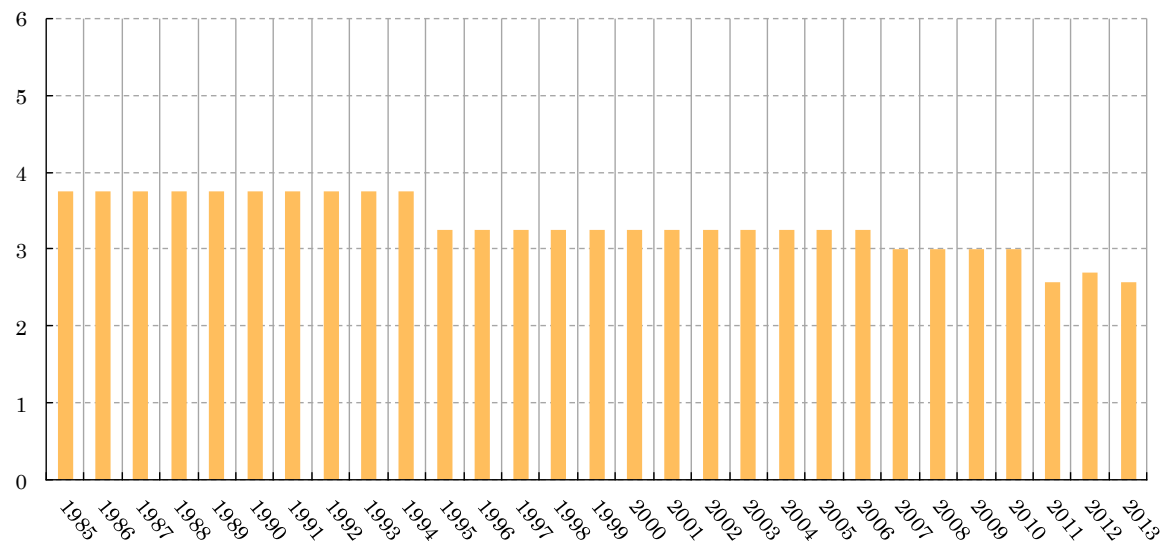

Fuente: OCDE.

Gráfico 3. España: Protección de los contratos indefinidos contra despidos individuales y colectivos. Versión 2 del indicador a 1 de Enero.

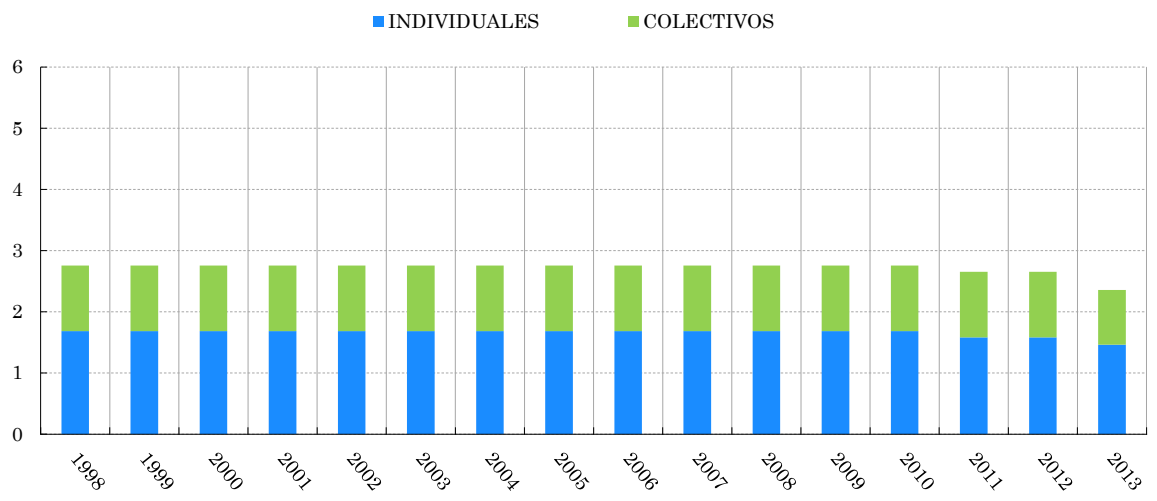

Fuente: OCDE.

De la revisión de las series temporales del indicador de la OCDE para España se desprende que, sin entrar en la cuestión del cumplimiento efectivo de la legislación y las prácticas judiciales (las cuales están jugando un papel de gran relevancia en la aplicación de las 
últimas reformas en España ${ }^{6}$, hay un conjunto de cambios normativos que parecen asimismo bastante relevantes y que no se reflejan en dichas series, como son la bonificación por parte del FOGASA de 8 días en el coste de despido objetivo, en vigor desde la reforma de 2010, las modificaciones en la definición de las circunstancias económicas que pueden motivar el despido, introducidas en 1995, 2010 y 2012, la regulación de los salarios de tramitación (y el procedimiento de "despido exprés" introducido en 2002 y ampliado en 2006) ni su posterior eliminación en 2012 o la supresión de la autorización administrativa realizada con la reforma de 2012. Además, entre las limitaciones de los indicadores de la OCDE, una de las que puede ser más importantes en el caso español es que no se tengan en cuenta las reformas parciales, lo que impide que se reflejen en dichos indicadores algunos cambios sustanciales de la regulación del despido, como son los que han afectado a los contratos indefinidos de fomento o la extensión del período de prueba en el contrato de "emprendedores".

\section{Los flujos del empleo asalariado}

Los flujos de entrada y salida del empleo asalariado (y de transiciones de empleo a empleo) que se analizan en este trabajo se han obtenido a partir de los datos de la EPA para el período comprendido entre el primer trimestre de 2005 y el primer trimestre de 2015. Dichos flujos se definen aquí en términos de transiciones trimestrales; es decir que, para un trimestre determinado, se considera que se ha producido una contratación cuando la antigüedad de un trabajador en la empresa es igual o inferior a tres meses. De igual modo, se considera que se ha producido una separación del empleo cuando la situación laboral del trabajador en el trimestre anterior era la de asalariado y en el trimestre actual es de ocupado no asalariado, desempleado o inactivo, o bien, en el caso de transiciones de empleo a empleo, la antigüedad del trabajador en la empresa es igual o inferior a tres meses ${ }^{7}$. Además de la información necesaria para definir los flujos (situación laboral y antigüedad en la empresa), la EPA ofrece otra información que permite

\footnotetext{
6 "La Audiencia Nacional anula la gran mayoría de los ERE en 2014", (Expansión, 19 de diciembre de 2014).

${ }^{7}$ En el primero de los análisis de regresión que se describen en la sección 4 se utilizan transiciones en términos interanuales, es decir definidas según la situación del trabajador en el mismo trimestre del año anterior. Aquí se toma como referencia el primer trimestre de cada año, ya que para 2015 solo se disponía hasta dicho período en el momento de efectuar los cálculos.
} 
clasificarlos por tipo de contrato y rama de actividad, si bien esta última desagregación solo es libremente accesible al nivel de un dígito (diez ramas de actividad).

Para cada trimestre y tipo de flujo (contrataciones, separaciones y transiciones de empleo a empleo), se ha calculado, el porcentaje que representa el número de transiciones respecto al stock de procedencia (lo que podríamos denominar probabilidad de transición). El gráfico 4 presenta dichas ratios para el total de contrataciones y separaciones y su descomposición por tipo de contrato. Las reformas laborales no parecen haber afectado a las probabilidades de contratación ni de separación, que muestran una cierta estabilidad desde el comienzo de la crisis.

Tampoco se aprecia un efecto significativo de las reformas en la probabilidad de contratación de desempleados, que solo detuvo su caída a partir de 2013, recuperándose ligeramente en 2014 (gráfico 5). Las probabilidades de transición de empleo a empleo se han mantenido notablemente por debajo de su nivel pre-crisis, mientras que la probabilidad de perder el empleo pasando al desempleo se ha mantenido alta, en torno al $5 \%$, sin que tampoco se aprecie efecto sustancial de las reformas laborales, salvo en el caso de los contratos indefinidos a partir del segundo trimestre de 2014.

\section{Gráfico 4. Probabilidad de transición en el empleo asalariado. Flujos en porcentaje de la población del grupo del que proceden.}
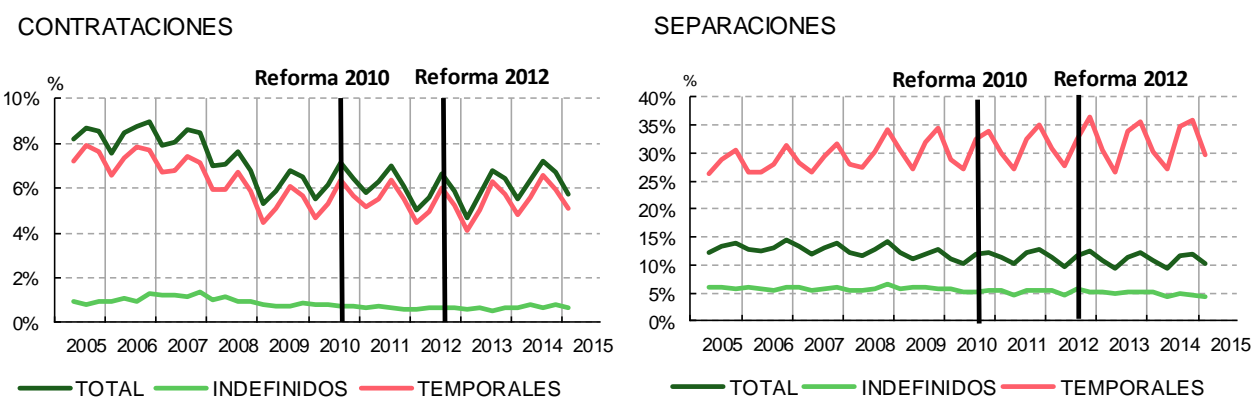

Fuente: Instituto Nacional de Estadística y elaboración propia 
En el gráfico 6 se presenta un mayor detalle sobre las transiciones de empleo a empleo. Se observa que las probabilidades de tránsito se redujeron drásticamente en 2008 y 2009, sin que se hayan visto afectadas por las reformas. Solo desde mediados de 2014 se observa una cierta recuperación, principalmente de los contratos temporales dentro del mismo sector de actividad (panel superior derecho). En el panel inferior derecho se observa además que esta recuperación se debe sobre todo a transiciones de un contrato temporal a otro. Por otro lado, las diferencias en términos de probabilidades de transición entre contratos temporales e indefinidos son amplias, de forma que en el primer trimestre de 2015, la probabilidad de tránsito de los primeros a otro empleo del mismo sector era unas 20 veces mayor que la de los últimos (paneles superiores del gráfico 6). Incluso la probabilidad de pasar de un contrato temporal a otro indefinido en el mismo sector (panel inferior izquierdo) duplica en dicho trimestre a la del movimiento en sentido contrario (de indefinido a temporal en el mismo sector; véase panel central derecho). En todos los casos, son más probables las transiciones dentro del mismo sector ${ }^{8}$.

\footnotetext{
8 Como señalan Bassanini y Garnero (2013), esto puede ser indicativo de la segmentación sectorial del mercado de trabajo, posiblemente como consecuencia de la acumulación de capital humano específico para cada sector.
} 


\section{Gráfico 5. Probabilidad de contratación y de separación del empleo. Flujos en porcentaje de la población del grupo del que proceden.}

CONTRATACIONES INDEFINIDAS Y TEMPORALES

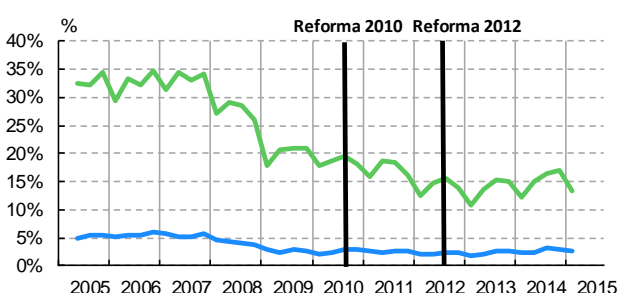

DESDE EL EMPLEO

DESDE EL DESEMPLEO

CONTRATACIONES INDEFINIDAS

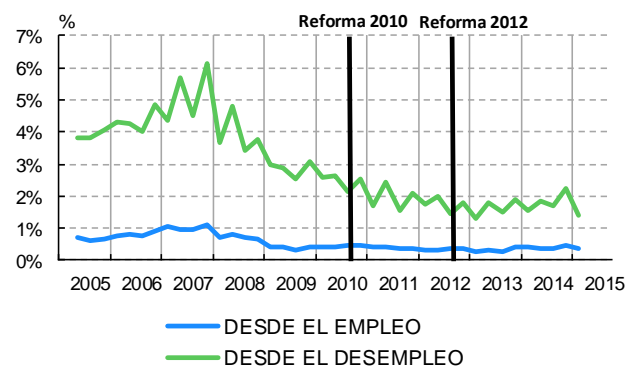

CONTRATACIONES TEMPORALES

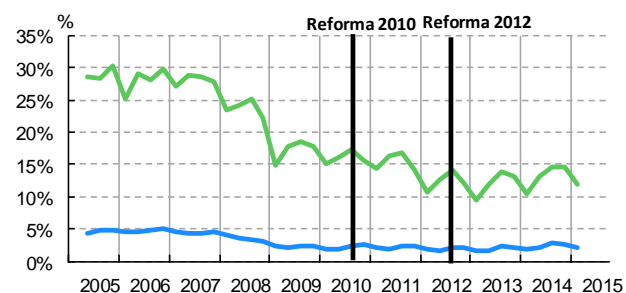

DESDE EL EMPLEO

DESDE EL DESEMPLEO
SEPARACIONES DEL EMPLEO INDEFINIDO Y TEMPORAL

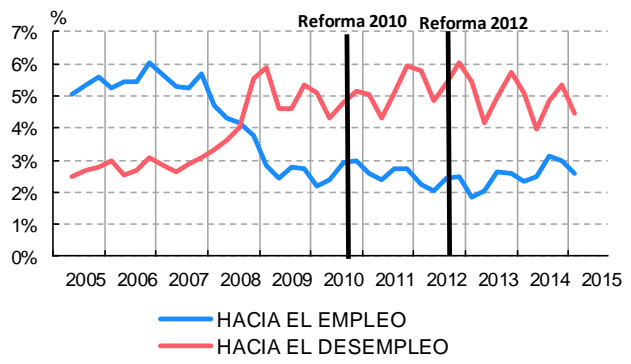

SEPARACIONES DEL EMPLEO INDEFINIDO

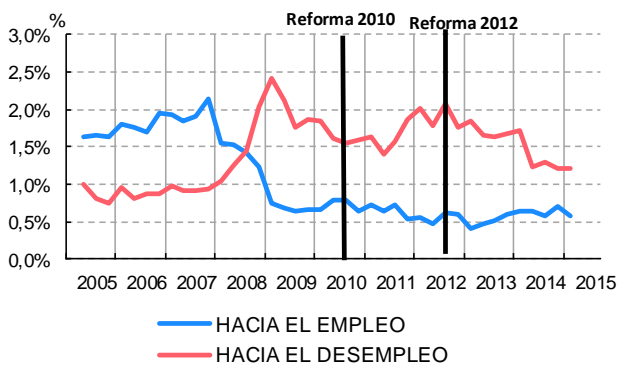

SEPARACIONES DEL EMPLEO TEMPORAL

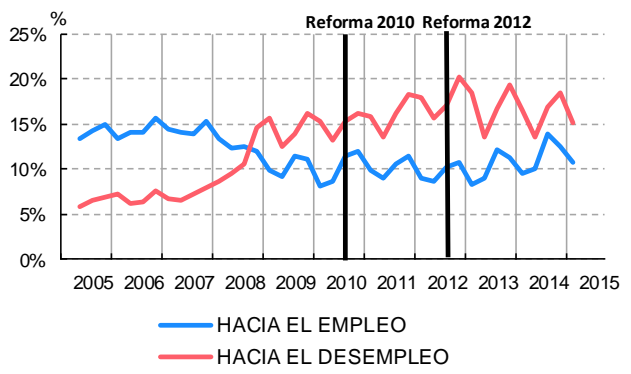

Fuente: Instituto Nacional de Estadística y elaboración propia 


\section{Gráfico 6. Probabilidad de transición de empleo a empleo. Flujos en porcentaje de la población del grupo del que proceden.}

DESDE CONTRATOS INDEFINIDOS

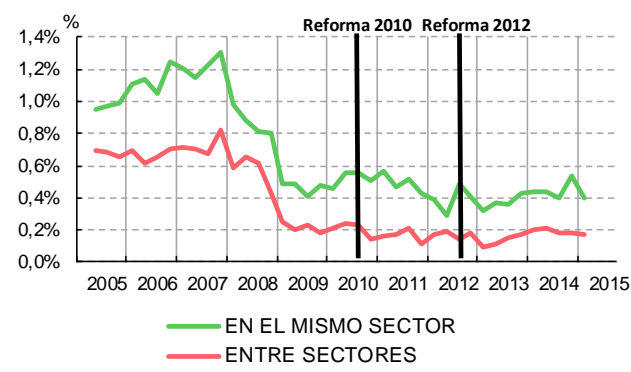

DE INDEFINIDOS A INDEFINIDOS

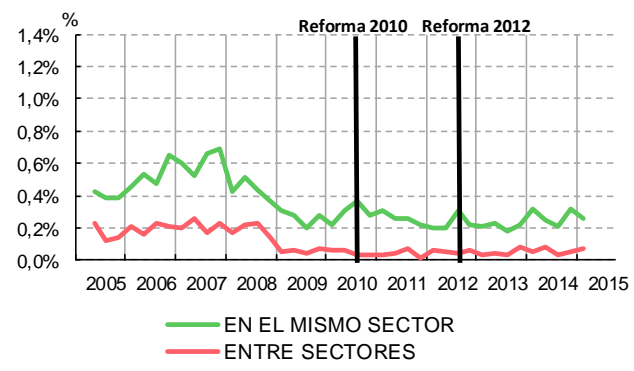

DE TEMPORALES A INDEFINIDOS

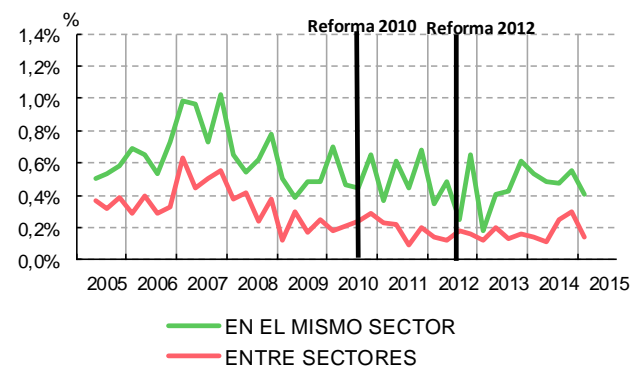

DESDE CONTRATOS TEMPORALES

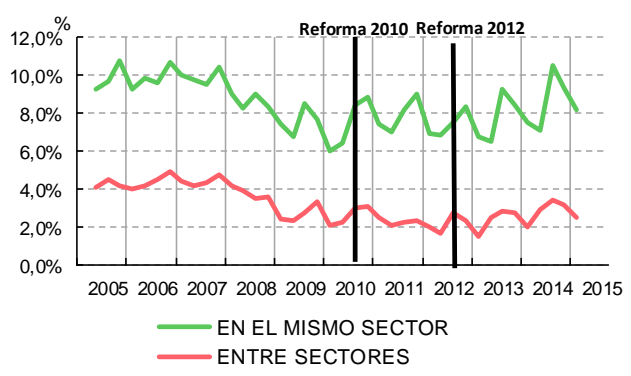

DE INDEFINIDOS A TEMPORALES

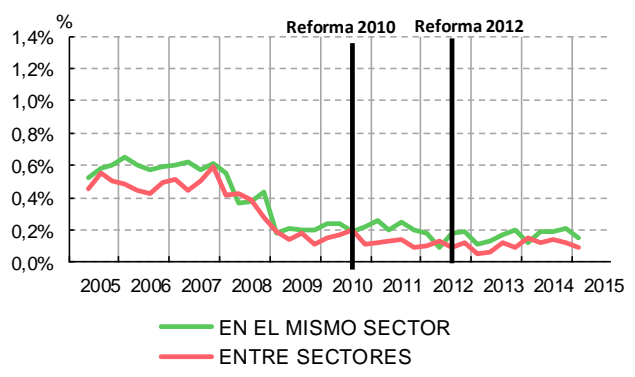

DE TEMPORALES A TEMPORALES

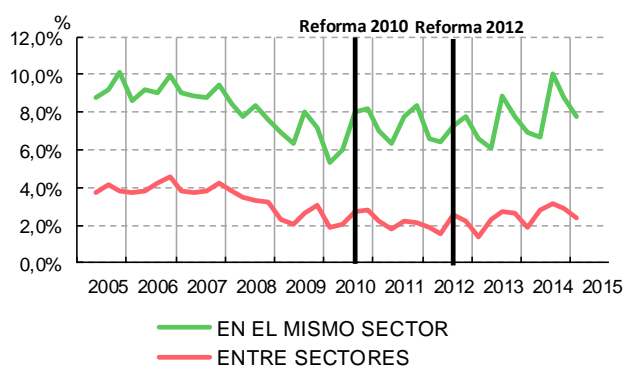

Fuente: Instituto Nacional de Estadística y elaboración propia

El análisis empírico que se aborda en la sección siguiente toma de la EPA, además de la información mencionada respecto al cálculo de flujos, otra sobre características demográficas y personales: género, 
edad, nacionalidad, nivel de estudios y, en el caso de los desempleados, tiempo de búsqueda de empleo y si perciben prestación o subsidio de desempleo. Los datos sobre instituciones del mercado de trabajo o de productos proceden de la OCDE. En el apéndice 1 se presentan los estadísticos descriptivos de las principales variables utilizadas y se describen las fuentes de forma más detallada. En particular, se consideran dos indicadores de protección del empleo: el de protección de los contratos indefinidos contra despidos individuales y colectivos (EPRC, versión 2) y el de restricción de los contratos temporales (EPT, versión 1 ).

\section{Análisis empírico}

Como primera aproximación a la medición del impacto de las reformas de la legislación de protección del empleo sobre los flujos descritos en la sección anterior, y tratando de explotar la información que la EPA ofrece a nivel sectorial, se estima un modelo lineal con la siguiente especificación:

$F_{t}^{i}=\beta \cdot X_{t}^{i}+\gamma \cdot E P L_{t}+\delta \cdot C_{t}^{i}+\lambda \cdot \Delta_{14} P I B_{t}+\eta_{i}+\eta_{t}+\varepsilon_{t}^{i}(1)$

donde $F_{t}^{i}$ es, para cada tipo de flujo, el porcentaje que representa el número de transiciones interanuales ${ }^{9}$ en el primer trimestre del año $t$ respecto al volumen de empleo asalariado en el sector $i, E P L_{t}$ es el valor del correspondiente indicador de protección del empleo a 1 de Enero del año $t+1, X_{t}^{i}$ y $C_{t}^{i}$ son vectores de control con las variables institucionales y de características demográficas y personales de los trabajadores del sector $i, \Delta_{14} P I B_{t}$ es la tasa de variación interanual del PIB real en el primer trimestre del año $t$, como variable de control del ciclo económico y $\varepsilon_{t}^{i}$ es el término de error, en tanto que $\eta_{i}$ y $\eta_{t}$ representan los efectos fijos de sector y año, respectivamente. Los vectores de parámetros a estimar son $\beta$ y $\delta$, además de los parámetros $\lambda$ y $\gamma$, siendo este último de especial interés. Los controles por características de los trabajadores del sector incluyen sexo (porcentaje de mujeres), edad (porcentajes de

9 El motivo para calcular así las transiciones es que los indicadores de protección del empleo son de frecuencia anual. Alternativamente, se han utilizado, por un lado, medias anuales de los flujos intertrimestrales y, por otro, estos últimos directamente, en este caso repitiendo en cada trimestre el dato anual de las variables institucionales. Los resultados no difieren sustancialmente de los que aquí se presentan. 
trabajadores de entre 16 y 29 años y de entre 45 y 64 años), nacionalidad (porcentaje de extranjeros), nivel de estudios (porcentajes de trabajadores con estudios primarios y de aquellos con estudios medios) y el porcentaje de trabajadores por cuenta propia. Las variables institucionales utilizadas (véase el apéndice 1) son la generosidad del sistema de prestaciones por desempleo, medido como producto de la tasa media de sustitución y la tasa de cobertura, la tasa de afiliación sindical, la cuña fiscal y un indicador de regulación en el mercado de productos. Dado que la última actualización disponible de los indicadores de protección del empleo se refiere al 1 de enero de 2013, el período utilizado para la estimación es 2005-2012.

Los cuadros 2 y 3 presentan los resultados de la estimación por MCO, con errores estándar robustos agrupados por rama de actividad. Solo las contrataciones totales y las transiciones de empleo a empleo totales y entre sectores presentan un coeficiente significativo (de signo negativo en cualquier caso) al 99\% de confianza para el indicador de protección contra despidos individuales y colectivos y solo las transiciones de empleo a empleo que implican un cambio de sector muestran un coeficiente (también negativo) con dicho grado de significatividad en el caso del indicador de restricción de la contratación temporal. Respecto al primero de estos indicadores, los coeficientes para las transiciones de empleo a empleo de los trabajadores con contrato temporal en el empleo de origen, por un lado, y para las de aquellos con contrato indefinido en el empleo de origen, por otro, también son significativos (y siempre de signo negativo) al $95 \%$ y al $90 \%$ de confianza, respectivamente. También es significativo al $90 \%$ de confianza (e igualmente de signo negativo) el coeficiente de dicho indicador en el caso de las transiciones totales. Las separaciones totales tienen también un coeficiente negativo en el indicador de despidos y las salidas del empleo al desempleo positivo, aunque no es significativo en ninguno de los dos casos. Estos resultados coinciden en parte con los de otros trabajos que estiman modelos similares (por ejemplo, Bassanini y Garnero, 2013), si bien resulta llamativa la ausencia de significatividad, en la mayoría de los casos, de la variable de control del ciclo económico ${ }^{10}$.

\footnotetext{
10 Parte del efecto del ciclo económico podría venir recogido por las variables temporales (dummies de año), por lo que se han estimado las regresiones excluyendo dichas variables, con resultados similares.
} 
Cuadro 2. Variables institucionales y flujos del empleo asalariado 2005-2012 (a) (b)

\begin{tabular}{|c|c|c|c|c|c|c|c|c|c|c|}
\hline & \multicolumn{2}{|c|}{$\begin{array}{c}\text { Transiciones } \\
\text { totales }\end{array}$} & \multicolumn{2}{|c|}{$\begin{array}{c}\text { Contrataciones } \\
\text { totales }\end{array}$} & \multicolumn{2}{|c|}{$\begin{array}{c}\text { Contrataciones de } \\
\text { desempleados }\end{array}$} & \multicolumn{2}{|c|}{$\begin{array}{c}\text { Separaciones } \\
\text { totales }\end{array}$} & \multicolumn{2}{|c|}{$\begin{array}{l}\text { Salidas al } \\
\text { desempleo }\end{array}$} \\
\hline \multicolumn{11}{|l|}{ INDICADORES DE PROTECCIÓN DEL EMPLEO } \\
\hline EPRC versión $2(\mathrm{c})$ & $-4,29 * * *$ & - & $-3,33 *$ & - & $-0,54$ & - & $-0,96$ & - & 0,84 & - \\
\hline EPT versión $1(\mathrm{~d})$ & - & $-1,59$ & - & $-1,10$ & - & 0,21 & - & $-0,49$ & - & $-0,18$ \\
\hline \multicolumn{11}{|l|}{ CONTROLES INSTITUCIONALES } \\
\hline Prestaciones por desempleo (e) & $0,26 * *$ & 0,07 & $0,21 \quad * *$ & 0,07 & 0,00 & 0,00 & 0,06 & 0,01 & $-0,15 \quad * * *$ & $-0,13 * *$ \\
\hline Afiliación sindical & $-1,01$ & $-0,76$ & $-0,59$ & $-0,39$ & $0,39 * *$ & $0,42 *$ & $-0,43$ & $-0,37$ & $0,91 * *$ & $0,86 * *$ \\
\hline Cuña fiscal & $-0,58$ & $-0,54$ & $-0,04$ & 0,01 & $0,52 *$ & $0,59 *$ & $-0,53$ & $-0,55$ & 0,28 & 0,20 \\
\hline Regulación del mercado de productos & $-0,20$ & $-0,22$ & 0,15 & 0,13 & 0,00 & $-0,01$ & $-0,34$ & $-0,35$ & $-0,25$ & $-0,25$ \\
\hline CRECIMIENTO DEL PIB REAL (f) & 0,12 & 0,11 & 0,00 & $-0,01$ & $-0,07 * * *$ & $-0,06 * * *$ & 0,12 & 0,12 & $0,10 * *$ & 0,10 \\
\hline DUMMIES POR SECTOR DE ACTIVIDAD & Sí & Sí & Sí & Sí & Sí & Sí & Sí & Sí & Sí & Sí \\
\hline DUMMIES POR AÑO & Sí & Sí & Sí & Sí & Sí & Sí & Sí & Sí & Sí & Sí \\
\hline CARACTERÍSTICAS DE LOS TRABAJADORES (g) & Sí & Sí & Sí & Sí & Sí & Sí & Sí & Sí & Sí & Sí \\
\hline \multicolumn{11}{|l|}{ PRO MEMORIA } \\
\hline Número de observaciones & 80 & 80 & 80 & 80 & 80 & 80 & 80 & 80 & 80 & 80 \\
\hline $\mathrm{R}^{2}$ & 0,8859 & 0,8816 & 0,8073 & 0,7960 & 0,7407 & 0,8446 & 0,8447 & 0,8441 & 0,7583 & 0,7567 \\
\hline
\end{tabular}

Fuente: Elaboración propia.

a. Regresiones MCO de flujos definidos en términos interanuales (comparando las situaciones laborales en el primer trimestre de cada año respecto al mismo trimestre del año anterior) y como porcentajes del volumen de empleo asalariado total.

b. Errores standard robustos (agrupados por ramas de actividad). Los símbolos *, ** y *** denotan significatividad al 99\%, al 95\% y al $90 \%$, respectivamente.

c. Valores a 1 de Enero del año $t+1$ para el indicador de despidos individuales y colectivos.

d. Valores a 1 de Enero del año $t+1$ para el indicador de contratos temporales.

e. Producto de la tasa media de sustitución y de la tasa de cobertura.

f. Tasa de variación interanual

g. Porcentaje de mujeres, de trabajadores con edades entre 16 y 29 años y entre 45 y 64 años, de extranjeros, de trabajadores con estudios primarios y con estudios medios y de trabajadores por cuenta propia. 
Cuadro 3. Variables institucionales y flujos de empleo a empleo 2005-2012 (a) (b)

\begin{tabular}{|c|c|c|c|c|c|c|c|c|c|c|}
\hline & \multicolumn{2}{|c|}{$\begin{array}{c}\text { Totales del } \\
\text { empleo } \\
\text { asalariado }\end{array}$} & \multicolumn{2}{|c|}{ En el mismo sector } & \multicolumn{2}{|c|}{ Entre sectores } & \multicolumn{2}{|c|}{$\begin{array}{c}\text { Contratos } \\
\text { indefinidos (h) }\end{array}$} & \multicolumn{2}{|c|}{$\begin{array}{c}\text { Contratos } \\
\text { temporales (h) }\end{array}$} \\
\hline \multicolumn{11}{|l|}{ INDICADORES DE PROTECCIÓN DEL EMPLEO } \\
\hline EPRC versión 2 (c) & $-2,10 *$ & - & $-0,78$ & - & $-1,32 *$ & - & $-0,81 * * *$ & - & $-1,28 * *$ & - \\
\hline EPT versión $1(\mathrm{~d})$ & - & $-0,86$ & - & $-1,59$ & - & $-0,94$ & - & $-0,34$ & - & $-0,53$ \\
\hline \multicolumn{11}{|l|}{ CONTROLES INSTITUCIONALES } \\
\hline Prestaciones por desempleo (e) & $0,16 *$ & 0,06 & $0,09 * *$ & $0,09 * *$ & $0,06 *$ & $-0,01$ & 0,04 & 0,00 & $0,12 *$ & 0,06 \\
\hline Afiliación sindical & $-0,91 *$ & $-0,79 *$ & $-0,46 * * *$ & $-0,52 * * *$ & $-0,46 * *$ & $-0,38 * *$ & $-0,24$ & $-0,20$ & $-0,67 * *$ & $-0,60 * *$ \\
\hline Cuña fiscal & $-0,54 *$ & $-0,53 * *$ & $-0,19$ & $-0,29 * * *$ & $-0,35 * *$ & $-0,40 *$ & $-0,16$ & $-0,16$ & $-0,38 * *$ & $-0,37 * * *$ \\
\hline Regulación del mercado de productos & 0,12 & 0,11 & 0,09 & 0,09 & 0,03 & 0,02 & 0,05 & 0,04 & 0,07 & 0,06 \\
\hline CRECIMIENTO DEL PIB REAL (f) & 0,04 & 0,03 & 0,04 & 0,03 & 0,00 & $-0,01$ & 0,00 & 0,00 & 0,04 & 0,03 \\
\hline DUMMIES POR SECTOR DE ACTIVIDAD & Sí & Sí & Sí & Sí & Sí & Sí & Sí & Sí & Sí & Sí \\
\hline DUMMIES POR AÑO & Sí & Sí & Sí & Sí & Sí & Sí & Sí & Sí & Sí & Sí \\
\hline CARACTERÍSTICAS DE LOS TRABAJADORES (g) & Sí & Sí & Sí & Sí & Sí & Sí & Sí & Sí & Sí & Sí \\
\hline \multicolumn{11}{|l|}{ PRO MEMORIA } \\
\hline Número de observaciones & 80 & 80 & 80 & 80 & 80 & 80 & 80 & 80 & 80 & 80 \\
\hline $\mathrm{R}^{2}$ & 0,8384 & 0,8286 & 0,7835 & 0,7837 & 0,6966 & 0,6784 & 0,5976 & 0,5818 & 0,8084 & 0,8035 \\
\hline
\end{tabular}

Fuente: Elaboración propia.

a. Regresiones MCO de flujos definidos en términos interanuales (comparando las situaciones laborales en el primer trimestre de cada año respecto al mismo trimestre del año anterior) y como porcentajes del volumen de empleo asalariado total.

b. Errores standard robustos (agrupados por ramas de actividad). Los símbolos *** y *** denotan significatividad al 99\%, al 95\% y al 90\%, respectivamente.

c. Valores a 1 de Enero del año $t+1$ para el indicador de despidos individuales y colectivos.

d. Valores a 1 de Enero del año $t+1$ para el indicador de contratos temporales.

e. Producto de la tasa media de sustitución y de la tasa de cobertura.

f. Tasa de variación interanual.

g. Porcentaje de mujeres, de trabajadores con edades entre 16 y 29 años y entre 45 y 64 años, de extranjeros, de trabajadores con estudios primarios

y con estudios medios y de trabajadores por cuenta propia.

h. En la situación de partida. 
Cuadro 4. Estimación del impacto de las reformas del despido sobre los flujos de empleo asalariado (a) (b). Porcentaje del volumen de empleo asalariado total.

\begin{tabular}{|c|c|c|c|c|c|c|c|c|c|}
\hline & \multirow{2}{*}{\multicolumn{3}{|c|}{ Contrataciones }} & \multicolumn{6}{|c|}{ Salidas } \\
\hline & & & & \multirow[b]{2}{*}{ Totales } & & \multicolumn{4}{|c|}{ A otro empleo asalariado } \\
\hline & $\begin{array}{c}\text { Transiciones } \\
\text { totales }\end{array}$ & Totales & \multirow[t]{2}{*}{$\begin{array}{c}\text { De } \\
\text { desempleados }\end{array}$} & & $\begin{array}{c}\text { Al } \\
\text { desempleo }\end{array}$ & Totales & $\begin{array}{c}\text { En el mismo } \\
\text { sector }\end{array}$ & $\begin{array}{c}\text { En otro } \\
\text { sector }\end{array}$ & \\
\hline \multicolumn{9}{|c|}{ CONTRATOS INDEFINIDOS Y TEMPORALES } & \\
\hline REFORMA DE 2010 & $0,44 \% \quad * * *$ & $0,34 \% *$ & $0,06 \%$ & $0,10 \%$ & $-0,09 \%$ & $0,21 \%$ * & $0,08 \%$ & $0,13 \%$ & * \\
\hline REFORMA DE 2012 & $1,28 \%$ & $0,99 \%$ * & $0,16 \%$ & $0,28 \%$ & $-0,25 \%$ & $0,62 \%$ * & $0,23 \%$ & $0,39 \%$ & \\
\hline \multicolumn{10}{|c|}{ CONTRATOS INDEFINIDOS (c) } \\
\hline REFORMA DE 2010 & $0,15 \%$ & $0,16 \% * *$ & $0,04 \%$ & $-0,01 \%$ & $-0,03 \%$ & $0,08 \% * * * *$ & $0,03 \%$ & $0,05 \%$ & *** \\
\hline REFORMA DE 2012 & $0,43 \%$ & $0,46 \% * *$ & $0,13 \%$ & $-0,03 \%$ & $-0,08 \%$ & $0,24 \% * * * *$ & $0,10 \%$ & $0,14 \%$ & ** \\
\hline \multicolumn{10}{|c|}{ CONTRATOS TEMPORALES (c) } \\
\hline REFORMA DE 2010 & $0,29 \%$ & $0,18 \% * * *$ & $0,01 \%$ & $0,11 \%$ & $-0,06 \%$ & $0,13 \%$ *** & $0,05 \%$ & $0,09 \%$ & $* *$ \\
\hline REFORMA DE 2012 & $0,84 \%$ & $0,53 \% \quad * * *$ & $0,04 \%$ & $0,31 \%$ & $-0,17 \%$ & $0,38 \%$ ** & $0,13 \%$ & $0,25 \%$ & $* *$ \\
\hline \multicolumn{10}{|c|}{ DE INDEFINIDO A INDEFINIDO } \\
\hline REFORMA DE 2010 & - & - & - & - & - & $0,05 \% * * *$ & $0,04 \%$ & $0,02 \%$ & \\
\hline REFORMA DE 2012 & - & - & - & - & - & $0,15 \% \quad * * *$ & $0,11 \%$ & $0,05 \%$ & \\
\hline \multicolumn{10}{|c|}{ DE INDEFINIDO A TEMPORAL } \\
\hline REFORMA DE 2010 & - & - & - & - & - & $0,03 \%$ & $0,00 \%$ & $0,03 \%$ & $* * * *$ \\
\hline REFORMA DE 2012 & - & - & - & - & - & $0,09 \%$ & $-0,01 \%$ & $0,09 \%$ & $* * * * *$ \\
\hline \multicolumn{10}{|c|}{ DE TEMPORAL A INDEFINIDO } \\
\hline REFORMA DE 2010 & - & - & - & - & - & $0,05 \%$ * & $0,03 \%$ & $0,02 \%$ & *** \\
\hline REFORMA DE 2012 & - & - & - & - & - & $0,16 \%$ * & $0,10 \%$ & $0,06 \%$ & $* *$ \\
\hline \multicolumn{10}{|c|}{ DE TEMPORAL A TEMPORAL } \\
\hline REFORMA DE 2010 & - & - & - & - & - & $0,08 \%$ & $0,01 \%$ & $0,07 \%$ & $* *$ \\
\hline REFORMA DE 2012 & - & - & - & - & - & $0,23 \%$ & $0,03 \%$ & $0,19 \%$ & $* *$ \\
\hline
\end{tabular}

Fuente: Elaboración propia.

a. De acuerdo con los cambios en el indicador de despidos individuales y colectivos (EPRC versión 2).

b. Los símbolos * $* *$ y *** denotan significatividad al $99 \%$, al $95 \%$ y al $90 \%$, respectivamente.

c. En la situación de partida. 
El impacto de las reformas del despido en 2010 y 2012, calculado de acuerdo con las estimaciones anteriores, se presenta en el cuadro 4 . La reforma de 2010 habría incrementado las contrataciones totales en una cifra equivalente al $0,3 \%$ de la población asalariada, en tanto que la de 2012 lo habría hecho en el 1\%; repartiéndose dicho aumento prácticamente por igual entre contratos indefinidos y temporales. El número de transiciones de empleo a empleo inducido por las reformas habría representado un $0,2 \%$ del empleo asalariado la de 2010 , y un 0,6\% la de 2012; del que casi dos tercios llevarían aparejado un cambio de sector, afectando a contratos temporales en similar proporción. El resto de transiciones presentan menos significatividad en términos estadísticos.

En cualquier caso, ha de tenerse en cuenta la escasa variabilidad temporal de los indicadores institucionales, y especialmente los de protección del empleo (gráficos 2 y 3), así como el hecho de que la desagregación sectorial disponible es solo a nivel de un dígito de la CNAE. Por otro lado, solo se dispone de un año para evaluar los efectos de la reforma de 2012. Además, también Bassanini y Garnero (2013) señalan que este tipo de especificación tiene el problema de que es difícil controlar por todas las instituciones que pueden estar correlacionadas con la variable de interés (en este caso la regulación de protección del empleo) y que dicha regulación puede ser endógena a la situación del mercado de trabajo. Finalmente, como se ha señalado en la sección 2, los indicadores de protección del empleo de la OCDE presentan en el caso español limitaciones importantes a la hora de recoger el conjunto de cambios normativos que sobre esta materia se implementaron con las reformas de 2010 y 2012.

Por todo ello, se utiliza una aproximación alternativa, más amplia ya que cuantifica no solo el efecto de los cambios regulatorios de la protección del empleo, sino el efecto global de las reformas, y que permite que el impacto de estas sobre los flujos del mercado de trabajo pueda ser no lineal. En concreto, se estiman, mediante modelos probit, las probabilidades de transitar entre el empleo asalariado y el desempleo, así como de un empleo a otro, controlando por características del trabajador (sexo, edad, nacionalidad y nivel de estudios) y del puesto de trabajo (sector de actividad, y, en su caso, tipo de contrato y antigüedad en la empresa). Además, se controla por estacionalidad y por el ciclo económico, medido por la tasa de variación 
intertrimestral del PIB real ajustado de estacionalidad ${ }^{11}$. Para estimar el impacto de las reformas sobre dichas probabilidades se ha utilizado el período completo (desde el primer trimestre de 2005 hasta el primer trimestre de 2015), incluyendo sendas variables artificiales (dummies) para recoger dicho impacto ${ }^{12}$. Así pues, el modelo estimado es:

$\operatorname{Prob}\left(T_{t}^{i}=1\right)=\beta \cdot D_{t}^{i}+\lambda \cdot \Delta_{11} P I B_{t}^{s a}+\tau \cdot \eta_{t r i m}+\psi_{1} \cdot \eta_{2010}+$
$\psi_{2} \cdot \eta_{2012}+\varepsilon_{t}^{i}$

donde $T_{t}^{i}$ es, para cada tipo de transición, una variable dicotómica que toma el valor 1 si el individuo $i$ efectúa esa transición (intertrimestral ${ }^{13}$ ) en el trimestre $t, D_{t}^{i}$ es el vector de control con las características del trabajador $i$ y de su puesto de trabajo, $\Delta_{11} P I B_{t}^{s a}$ es la tasa de variación intertrimestral del PIB real ajustado de estacionalidad en el trimestre $t$, $\eta_{\text {trim }}$ es un vector de dummies estacionales, en tanto que $\eta_{2010} \mathrm{y} \eta_{2012}$ son dummies que toman el valor 0 para los trimestres anteriores al de aprobación de la reforma y 1 para el trimestre en que se aprueba la reforma y los posteriores y $\varepsilon_{t}^{i}$ es el término de error. Se estiman los vectores de parámetros $\beta$ y $\tau$, y los parámetros $\lambda, \psi_{1}$ y $\psi_{2}$ siendo estos dos últimos los de principal interés. El individuo de referencia en la estimación de estos modelos es un hombre menor de 30 años, de nacionalidad española, con estudios superiores, con más de 3 años en el empleo y con un contrato indefinido en la rama de otros servicios. En el caso de las dummies estacionales la referencia es el primer trimestre.

11 Se ha realizado un análisis de sensibilidad utilizando variables alternativas de control del ciclo económico como el output gap, calculado según la metodología expuesta en Hernández de Cos et al. (2011), el empleo (medido por la tasa de variación intertrimestral de la serie de ajustada de estacionalidad de los afiliados a la Seguridad Social) y la tasa de paro. Utilizando el output gap como variable de ciclo, ya sea en solitario o en combinación con el empleo, los efectos estimados para la reforma de 2012 cambian su signo (y son significativos), respecto a las estimaciones que utilizan el PIB. Por último, si se utiliza la tasa de paro como variable de control del ciclo, todos los resultados cambian su signo (y son significativos), respecto a dichas estimaciones.

12 Las variables toman el valor 0 para los trimestres anteriores al de aprobación de la reforma y 1 para el trimestre en que se aprueba la reforma y los posteriores. Utilizando definiciones alternativas de estas dummiesse obtienen resultados similares.

13 Se han realizado, como prueba de robustez, estimaciones con transiciones interanuales utilizando datos de un solo trimestre para cada año. Solo se observan diferencias significativas en cuanto al efecto de la reforma de 2012 sobre las probabilidades de salir del empleo asalariado al desempleo, que pasaría a ser negativo. 
Los efectos estimados de las reformas para cada tipo de flujo y de contrato se presentan en el cuadro 5. En cuanto al signo, se observan grandes diferencias respecto a las estimaciones recogidas en el cuadro 4. De hecho, ambas aproximaciones solo coinciden en que las reformas habrían incrementado la tasa de rotación de los contratos temporales y, como consecuencia, del total, debido (parcialmente en el caso de la primera aproximación y totalmente en el caso de la segunda) al aumento de las separaciones del empleo temporal. Sin embargo, el signo que ambas estimaciones ofrecen para el impacto que las reformas hayan podido tener sobre el número de contrataciones (tanto totales como de desempleados), de salidas al desempleo y de transiciones de empleo a empleo es en casi todos los casos opuesto y solo coinciden en señalar un incremento de las transiciones de un contrato temporal a otro como consecuencia de la reforma de 2012 y de las contrataciones totales y temporales de desempleados a resultas de la reforma de 2010, aunque solo aparece como significativo este último y en la aproximación alternativa, con un impacto de 1,5 puntos porcentuales (cuadro 5). Junto a este, los mayores impactos estimados con dicha aproximación alternativa, serían la disminución (también del 1,5\%) de esas mismas contrataciones temporales de desempleados, inducida por la reforma de 2012 y el incremento en un $1,8 \%$ de las separaciones totales de trabajadores temporales debido a la reforma de 2010, impulsado por un aumento del $4,5 \%$ en las salidas al desempleo de este tipo de contratos. La reforma de 2012 también habría incrementado significativamente estos dos últimos flujos, aunque en menor cuantía $(0,7 \%$ y $1,2 \%$, respectivamente). 


\section{Cuadro 5. Estimación del impacto de las reformas laborales sobre la probabilidad de tránsito entre diferentes situaciones del empleo asalariado (a) (b)}

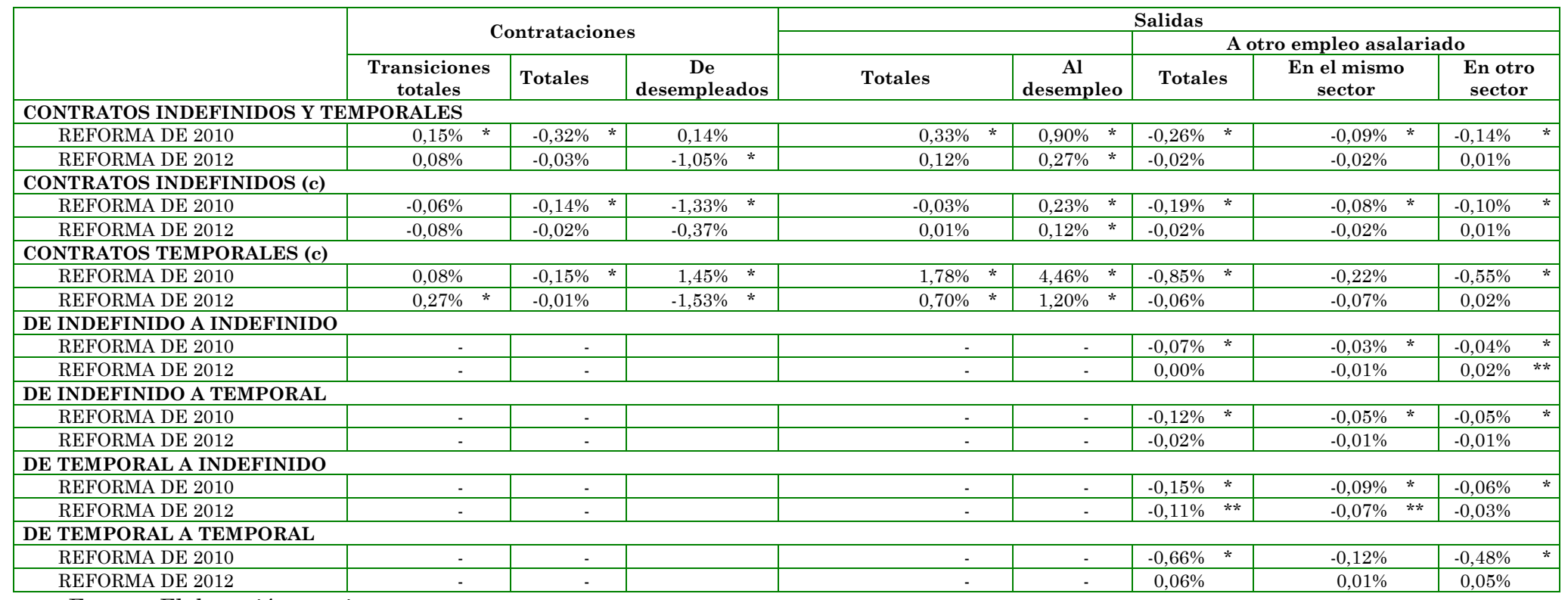

Fuente: Elaboración propia.

a. Los coeficientes muestran el efecto marginal de las reformas en pp y se obienen de regresiones probit que controlan por características personales (sexo, edad, nacionalidad y educación) y del puesto de trabajo (sector de actividad y, si procede, antigüedad y tipo de contrato), dummies estacionales y ciclo económico (tasa intertrimestral del PIB).

b. Los símbolos * ** y *** denotan significatividad al $99 \%$ y al $95 \%$, respectivamente.

c. En la situación de partida. 
Una visión complementaria de estos resultados se obtiene con la siguiente especificación del modelo probit :

$\operatorname{Prob}\left(T_{t}^{i}=1\right)=\beta \cdot D_{t}^{i}+\lambda \cdot \Delta_{11} P I B_{t}^{s a}+\tau \cdot \eta_{\text {trim }}+\psi \cdot \eta_{a}+\varepsilon_{t}^{i}(3)$

donde las dummies que tratan de captar el efecto de la reforma han sido sustituidas por un vector de dummies $\left(\eta_{a}\right)$,cada una de las cuales recoge un efecto temporal del año correspondiente (siendo 2005 el año de referencia) y donde el vector de características $D_{t}^{i}$ ha sido modificado, en el caso de las transiciones desde el desempleo al empleo, de forma que solo recoge las características individuales del desempleado, con dos variables adicionales: tiempo de búsqueda del empleo (la referencia es menos de 6 meses) y percepción o no de prestación o subsidio de desempleo (el individuo de referencia no es perceptor). El gráfico 7 muestra los efectos anuales sobre las probabilidades de transición entre el empleo asalariado y el desempleo. En consonancia con los resultados presentados en el cuadro 5, el panel izquierdo muestra un incremento en 2012 del efecto anual negativo sobre la probabilidad de transición desde el desempleo al empleo asalariado (dicho efecto se modera ligeramente en 2013 y 2014) ${ }^{14}$. Simétricamente, el panel derecho del gráfico recoge un incremento continuado desde 2008 hasta 2013 del efecto anual sobre la probabilidad de pasar del empleo asalariado al desempleo, sin que las reformas hayan alterado esa tendencia. Solo en 2014 se produjo un descenso de dicho efecto.

\footnotetext{
14 En 2010 también se observa un mayor efecto negativo (no así en 2011), lo que contrasta en parte con el coeficiente positivo, aunque no significativo, que presenta la dummy correspondiente a la reforma de ese año en la probabilidad de contratación de desempleados. Esta diferencia se puede deber a los cambios en el vector de control de características introducidos en este caso y a que la reforma se implementó en la parte final del año.
}

(C) Revista de Economía Laboral 


\section{Gráfico 7. Efectos anuales sobre las probabilidades de transición entre el empleo asalariado y el desempleo (a)}
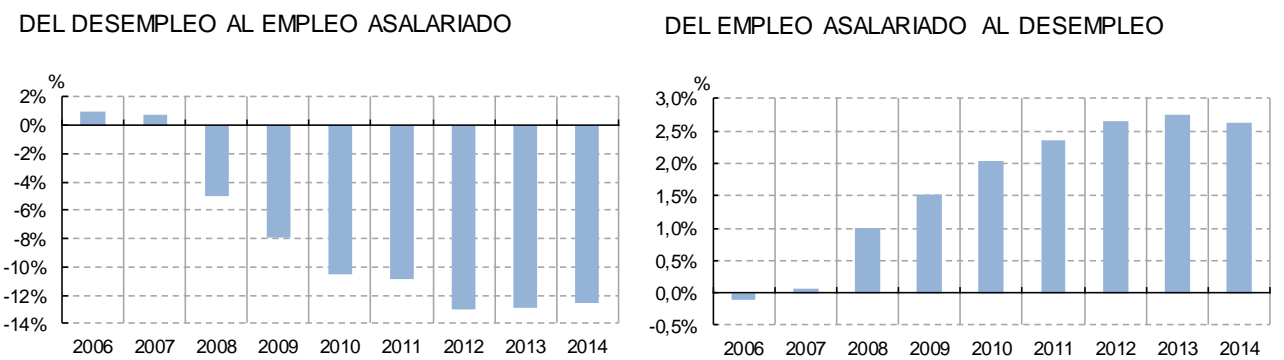

Fuente: Elaboración propia

a. La regresión incluye controles por características personales (sexo, edad, nacionalidad y educación) y, en el caso de las transiciones del empleo asalariado al desempleo, del puesto de trabajo (antigüedad, tipo de contrato y sector de actividad), duración del desempleo y percepción de prestación (en el caso de transiciones del desempleo asalariado), dummies estacionales y ciclo económico (tasa intertrimestral del PIB).

\section{Conclusiones}

En este artículo se analiza el impacto de las reformas laborales de 2010 y 2012, particularmente en lo que atañe a la legislación de protección del empleo, sobre los principales flujos del mercado de trabajo (las transiciones entre el desempleo y el empleo asalariado, y dentro de este). En el área de la protección del empleo, las reformas se reflejan en una disminución del grado de protección de los contratos indefinidos contra los despidos, tanto individuales como colectivos, al tiempo que se ha seguido facilitando la contratación temporal. Con todas las cautelas que implica un análisis de estas características, máxime teniendo en cuenta que se trata de reformas de largo alcance y el tiempo transcurrido desde su aprobación, no parece que las reformas en su conjunto hayan afectado de forma sustancial a las probabilidades de contratación ni de separación del empleo, lo que contrasta con los resultados de algunos de los estudios que han evaluado previamente los efectos de la reforma de 2012 (en particular, OCDE, 2013b). Tampoco se aprecia un efecto importante en la probabilidad de contratación de desempleados, que solo detuvo su caída a partir de 2013, recuperándose ligeramente en 2014. Las probabilidades de transición de empleo a 
empleo se han mantenido notablemente por debajo de su nivel precrisis, mientras que la probabilidad de perder el empleo pasando al desempleo se ha mantenido alta, sin que tampoco se aprecie efecto sustancial de las reformas laborales, salvo en el caso de los contratos indefinidos a partir del segundo trimestre de 2014.

Ambas reformas se plantean como uno de sus principales objetivos corregir la dualidad del mercado de trabajo; sin embargo, del análisis empírico realizado parece deducirse que habrían incrementado la tasa de rotación de los contratos temporales y, como consecuencia, del total, con un aumento de las separaciones del empleo temporal (también a diferencia de lo obtenido en OCDE, 2013b). Si el análisis se centra exclusivamente en los efectos de las reformas del despido, sí parece apreciarse un cierto incremento de las transiciones de empleo a empleo, principalmente las que han afectado a los contratos temporales y las que han llevado aparejado un cambio de sector.

En cualquier caso, ha de tenerse en cuenta la escasa variabilidad temporal de los indicadores de protección del empleo y que estos presentan en el caso español limitaciones importantes a la hora de recoger el conjunto de cambios normativos que sobre esta materia se implementaron con las reformas de 2010 y 2012. Por otro lado, la utilización de información sectorial más desagregada y de fuentes alternativas, como la Muestra Continua de Vidas Laborales, ofrecen posibles vías de mejora y contraste de las estimaciones aquí presentadas.

\section{Referencias}

Addison, J.T. y Teixeira, P. (2003). "The Economics of Employment Protection", Journal of Labor Research, Vol. 24, Issue 1, pp. 85-129.

Bassanini, A. y Garnero, A. (2013). "Dismissal Protection and Worker Flows in OECD countries: Evidence from Cross-country/Cross-industry Data", Labour Economics, Vol. 21, pp. 25-41.

Bertola, G., Boeri, T. y Cazes, S. (1999). Employment protection and labour market adjustment in OECD countries: evolving institutions and 
variable enforcement, Employment and Training Papers, 48, International Labour Office, Geneva.

Boeri, T. (2011). "Institutional reforms and dualism in european labor markets", en O. Ashenfelter y D. Card (eds.), Handbook of Labor Economics, vol. 4b, Elsevier, Amsterdam, 1173-1236.

Davis, S.J., Faberman, R.J. y Haltiwanger, J. (2006). "The Flow Approach to Labor Markets: New Data Sources and Micro-Macro Links", Journal of Economics Perspectives, Vol. 20, No. 3, pp. 3-26

Hernández de Cos, P., Izquierdo, M. y Urtasun, A. (2011). Una estimación del crecimiento potencial de la economía española, Documentos Ocasionales, n. ${ }^{\circ}$ 1104, Banco de España.

Izquierdo, M., Lacuesta, A. y Puente, S. (2013). "La reforma laboral de 2012: un primer análisis de algunos de sus efectos sobre el mercado de trabajo". Boletíneconómico, septiembre 2013, Banco de España.

Lazear, E. (1990). "Job security provisions and employment", The Quarterly Journal of Economics, 105(3), 699-726.

Ministerio de Empleo y Seguridad Social (2013). Informe de evaluación del impacto de la reforma laboral.

OCDE (2006). Employment Outlook, capítulo 3, Paris.

OCDE (2013a). Employment Outlook, capítulo 2, Paris.

OCDE (2013b). The 2012 Labour Market Reform In Spain: A Preliminary Assessment, http://www.oecd.org/els/emp/SpainLabourMarketReform-Report.pdf.

Pérez Infante, J.I. (2015). "Las reformas laborales en la crisis económica: su impacto económico", Ekonomiaz, 87, primer semestre 2015, pp. 247-281.

Pissarides, C. (2000). Equilibrium Unemployment Theory, segundaedición, MIT Press, Cambridge. Mass. 


\section{APÉNDICE 1. Variables institucionales de control y estadísticos descriptivos}

La generosidad del sistema de prestaciones por desempleo se mide como el producto de la tasa media de sustitución neta y la tasa de cobertura. La primera se obtiene como media de las tasas correspondientes a tres situaciones de renta $(67 \%, 100 \%$ y $150 \%$ del salario medio), doce situaciones familiares (solteros, con cónyuge dependiente o con ambos miembros de la pareja trabajando, en los tres casos sin hijos y con dos hijos; y en los seis casos, para familias elegibles o no para ayudas en vivienda o de asistencia social) y dos fases de la situación de desempleo (inicial y larga duración). La fuente es la base de datos de prestaciones y salarios, de la OCDE, con datos anuales para el período 20012013. La tasa de cobertura procede del Boletín de Estadísticas Laborales del Ministerio de Empleo y Seguridad Social, con datos anuales para el período 2004-2014.

La tasa de afiliación sindical se define como el porcentaje de asalariados miembros de un sindicato. La fuente es la base de datos de empleo, de la OCDE, que para España cubre el período 1980-2012.

La cuña fiscal es la diferencia entre el coste laboral para el empresario y el salario neto que recibe un trabajador que es el único perceptor de rentas en una pareja con dos hijos, y salario medio. La fuente es la base de datos de imposición sobre los salarios, de la OCDE, que cubre el período 2000-2014.

Finalmente, el indicador de regulación del mercado de productos, también procedente de la OCDE, proporciona indicadores que varían entre 0 y 6 , de menor a mayor grado de regulación, con datos anuales de 1975 a 2013 para los sectores de industrias de red (energía, transporte y comunicaciones), y quinquenales (1998, 2003, 2008 y 2013) para el comercio minorista. En este sector para los años intermedios se ha utilizado, alternativamente, una interpolación lineal o una 
repetición del último dato; sin prácticamente ninguna diferencia en los resultados.

En conjunto, la base de datos resultante con datos anuales (transiciones interanuales para los flujos laborales), utilizada en la primera aproximación empírica de este trabajo (ecuación 1) se compone de las variables cuyos estadísticos descriptivos se presentan en el cuadro A.1.

\section{Cuadro A.1. Estadísticos descriptivos. Base de datos anuales (modelo1)}

\begin{tabular}{|c|c|c|}
\hline Variable & Media & $\begin{array}{l}\text { Desviación } \\
\text { típica }\end{array}$ \\
\hline \multicolumn{3}{|l|}{ Variables institucionales } \\
\hline EPRC & 2,69 & 0,12 \\
\hline EPT & 2,92 & 0,25 \\
\hline Generosidad prestaciones por desempleo (\%) & 32,71 & 2,59 \\
\hline Cuña fiscal $(\%)$ & 33,70 & 0,97 \\
\hline Regulación del mercado de productos & 0,90 & 1,23 \\
\hline Afiliación sindical (\%) & 16,56 & 1,25 \\
\hline \multicolumn{3}{|l|}{ Flujos laborales (\% del empleo asalariado total) } \\
\hline Separaciones del empleo & 4,62 & 2,31 \\
\hline Contratos indefinidos & 2,08 & 0,94 \\
\hline Contratos temporales & 2,54 & 1,77 \\
\hline Contrataciones totales & 3,93 & 1,54 \\
\hline Contratos indefinidos & 0,85 & 0,45 \\
\hline Contratos temporales & 3,08 & 1,42 \\
\hline Transiciones de empleo a empleo (totales) & 1,70 & 1,04 \\
\hline En el mismo sector & 1,01 & 0,78 \\
\hline Entre sectores & 0,69 & 0,45 \\
\hline $\begin{array}{l}\text { Transiciones de empleo a empleo } \\
\text { (desde un contrato indefinido) }\end{array}$ & 0,58 & 0,34 \\
\hline En el mismo sector & 0,32 & 0,22 \\
\hline Entre sectores & 0,26 & 0,20 \\
\hline De indefinido a indefinido & 0,23 & 0,18 \\
\hline En el mismo sector & 0,15 & 0,13 \\
\hline Entre sectores & 0,09 & 0,10 \\
\hline De indefinido a temporal & 0,34 & 0,22 \\
\hline En el mismo sector & 0,18 & 0,15 \\
\hline Entre sectores & 0,17 & 0,14 \\
\hline $\begin{array}{l}\text { Transiciones de empleo a empleo } \\
\text { (desde un contrato temporal) }\end{array}$ & 1,13 & 0,85 \\
\hline En el mismo sector & 0,69 & 0,67 \\
\hline Entre sectores & 0,44 & 0,30 \\
\hline
\end{tabular}




\begin{tabular}{ccc}
\hline De temporal a indefinido & 0,18 & 0,15 \\
\hline En el mismo sector & 0,10 & 0,10 \\
\hline Entre sectores & 0,07 & 0,08 \\
\hline De temporal a temporal & 0,95 & 0,78 \\
\hline En el mismo sector & 0,59 & 0,63 \\
\hline Entre sectores & 0,36 & 0,25 \\
\hline Salidas al desempleo & 1,45 & 1,09 \\
\hline Contratos indefinidos & 0,58 & 0,46 \\
\hline Contratos temporales & 0,87 & 0,76 \\
\hline Contrataciones de desempleados & 1,21 & 0,72 \\
\hline Contratos indefinidos & 0,22 & 0,16 \\
\hline Contratos temporales & 0,99 & 0,65 \\
\hline Transiciones totales & 9,05 & 3,08 \\
\hline Contratos indefinidos & 3,15 & 0,87 \\
\hline Contratos temporales & 5,90 & 2,90 \\
\hline Características demográficas y personales (\%) & & \\
\hline Mujeres & 38,63 & 20,10 \\
\hline Edad: menores de 30 años & 18,77 & 5,40 \\
\hline Edad: 30-44 años & 42,52 & 9,39 \\
\hline Edad: mayores de 45 años & 38,71 & 9,71 \\
\hline Extranjeros & 12,54 & 7,63 \\
\hline Educación primaria & 17,52 & 12,81 \\
\hline Educación secundaria & 51,32 & 10,04 \\
\hline Educación superior & 31,16 & 16,34 \\
\hline Trabajadores por cuenta propia & 16,80 & 12,15 \\
\hline Tasa de variación del PIB (\%) & 0,72 & $-2,59$ \\
\hline
\end{tabular}

Fuente: Elaboración propia 